Check for updates

Cite this: Chem. Sci., 2019, 10, 5391

๑ All publication charges for this article have been paid for by the Royal Society of Chemistry

Received 22nd December 2018 Accepted 25th April 2019

DOI: $10.1039 / \mathrm{c} 8 \mathrm{sc} 05742 \mathrm{~g}$

rsc.li/chemical-science

\section{Oligoprolines guide the self-assembly of quaterthiophenes $\uparrow$}

\author{
Nellie A. K. Ochs, $\ddagger^{a}$ Urszula Lewandowska, $\ddagger^{a}$ Wojciech Zajaczkowski, (D) ${ }^{b}$ \\ Stefano Corra, (D) ${ }^{a}$ Stephan Reger, ${ }^{c}$ Andreas Herdlitschka, ${ }^{a}$ Sylvia Schmid, (D) c \\ Wojciech Pisula, (DD ${ }^{\text {bd }}$ Klaus Müllen, ${ }^{\star b}$ Peter Bäuerle (DD ${ }^{\star c}$ and Helma Wennemers (D) *a
}

Control over the molecular organization of $\pi$-conjugated oligothiophenes into different types of supramolecular assemblies is key to their use in organic electronics but difficult to achieve as these chromophores have a pronounced tendency to aggregate. Herein we show that oligoprolines, which do not self-assemble on their own, control the self-assembly of quaterthiophenes. Spectroscopic, microscopic, and diffraction studies with quaterthiophene-oligoproline conjugates revealed the formation of mono- or double-layered sheets or, alternatively, helically twisted ribbons - depending on the length of the oligoproline. The dimensions of the nanoscopic objects, which extend into the micrometer regime, correlate with the molecular dimensions of the quaterthiophene-oligoproline building blocks.

\section{Introduction}

Oligothiophenes are important compounds for the development of organic materials and have been used as active components in organic electronic devices. ${ }^{1,2}$ Their performance typically depends on their molecular organization in the solid state. $^{3-5}$ Supramolecular assembly has become a powerful approach for the bottom-up formation of oligothiophene-based nanostructures in solution and their subsequent integration into devices. ${ }^{6}$ In particular, conjugates of oligothiophenes with biocompatible auxiliaries such as peptides or carbohydrates resulted in a variety of well-ordered, often chiral, self-assembled nanostructures. ${ }^{7-12}$ Advanced nanostructures such as ribbons and fibrils have been achieved but typically require the use of synthetically tedious A-B-A motifs, in which the peptide (A) directs the $\pi-\pi$ stacking of the oligothiophene core (B) via hydrogen bonding., ${ }^{5,13-18}$ The resulting nanostructures adopt shapes that are dictated by the secondary structure of the auxiliary, e.g., $\beta$-sheet forming peptides. Peptidic auxiliaries that allow access to a larger variety of morphologies with facile

${ }^{a}$ Laboratory of Organic Chemistry, ETH Zürich, Vladimir-Prelog-Weg 3, 8093 Zürich, Switzerland. E-mail: Helma.Wennemers@org.chem.ethz.ch

${ }^{b}$ Max Planck Institute for Polymer Research, Ackermannweg 10, 55128 Mainz, Germany.E-mail: muellen@mpip-mainz.mpg.de

'Institute of Organic Chemistry II and Advanced Materials, University of Ulm, Germany. E-mail: peter.baeuerle@uni-ulm.de

${ }^{d}$ Department of Molecular Physics, Faculty of Chemistry, Lodz University of Technology, Zeromskiego 116, 90-924 Lodz, Poland

$\dagger$ Electronic supplementary information (ESI) available: Details on the synthesis and analytical data of all compounds, and additional spectral, microscopic, and diffraction data. See DOI: 10.1039/c8sc05742g

\$ These authors contributed equally. synthetic modifications would thus be valuable additions to the toolbox of self-assembly.

We have previously shown that a minimalistic (A-B) design based on conjugates of a single proline residue and a quaterthiophene moiety results in chiral, micellar assemblies. ${ }^{19,20} \mathrm{We}$ have also demonstrated that oligoproline scaffolds can guide the hierarchical self-assembly of sterically demanding electronpoor perylene monoimide (PMI) chromophores into a variety of unprecedented nanostructures. ${ }^{21-23}$ Oligoprolines adopt the lefthanded PP II helix in which every third residue is stacked on top of each other at a distance of $9 \AA$ (Fig. 1) ${ }^{24-27}$ Unlike $\alpha$-helices and $\beta$-sheets, this helix does not rely on stabilizing hydrogen bonds. As a result, six proline residues suffice to form PP II helices. Also in contrast to other peptides, short oligoprolines do not aggregate with each other and have therefore been used as inert molecular rulers and scaffolds. ${ }^{28,29}$ The studies on oligoproline-PMI conjugates furnished a remarkable outcome: two moieties that do not assemble on their own can, once conjugated, self-assemble into complex nanostructures. ${ }^{21-23}$ The question now arises whether oligoprolines can also control the self-assembly of electron-rich oligothiophenes, which have a pronounced intrinsic tendency toward $\pi-\pi$ stacking. ${ }^{6}$

Herein, we show that conjugation of a quaterthiophene moiety via a triazole linkage with oligoprolines allows for guiding the assembly of the chromophore. Variations of the length of the oligoproline moiety provided substantially different supramolecular assemblies, including nanostructured sheets, curls, or ribbons. We also present that the length of the oligoproline affects the assembly of the quaterthiophenes into parallel or antiparallel $\pi-\pi$ stacked aggregates and their further assembly into the higher ordered architectures. 


\section{Results and discussion}

\section{Design and synthesis of oligoproline-quaterthiophene conjugates}

To probe whether and to which extent oligoprolines influence the assembly of oligothiophenes, we connected a quaterthiophene with oligoprolines of different lengths. Specifically, we attached the quaterthiophene moiety to the second position of oligoproline 3-, 6-, 9-, and 12-mers via a triazole linker to obtain 2-5 (Fig. 1). Thus, within these conjugates the quaterthiophene is located at the same position with only the length of the oligoproline changing. The second proline residue was chosen as an anchoring site since this conjugation site had resulted in highly ordered assemblies with PMI-oligoproline conjugates. ${ }^{23}$ For the sake of comparison with these oligomers, we also linked the quaterthiophene to a monomeric azidoproline (Azp) residue to form conjugate 1 (Fig. 1).§
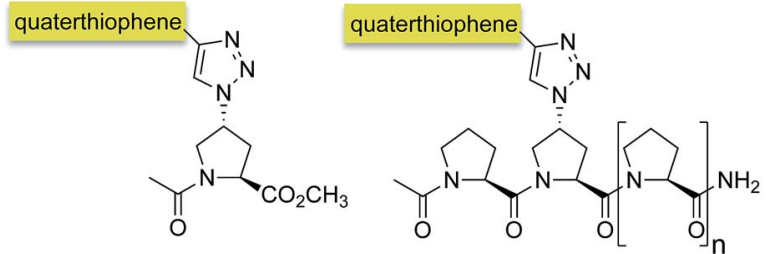

conjugates: 1

2: $n=1,3: n=4,4: n=7,5: n=10$

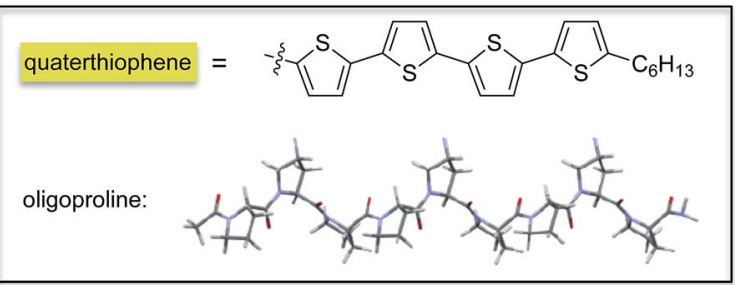

Fig. 1 Oligoproline-quaterthiophene conjugates 1-5.

The synthesis of conjugates 1-5 was accomplished by a combination of solid-phase peptide synthesis to furnish $(4 R)$ azidoproline containing oligoproline scaffolds followed by attachment of the quaterthiophene by $\mathrm{Cu}(\mathrm{I})$-catalyzed Huisgen's 1,3-cycloaddition with the alkyne-functionalized quaterthiophene. $\uparrow$

\section{Self-assembly of oligoproline-quaterthiophene conjugates 1- 5 in solution}

We first studied the self-assembly of the conjugates in solution. UV/Vis absorption spectra of 1-5 in THF, a common solvent for both the oligoproline and the $\pi$-system, are consistent with molecularly dissolved quaterthiophene, with $\lambda_{\max }=417 \pm 4 \mathrm{~nm}$ (Fig. 2, top, dashed lines). ${ }^{30}$ Upon addition of water to finally reach a ratio of THF : $\mathrm{H}_{2} \mathrm{O} 10: 90$ and annealing to $90{ }^{\circ} \mathrm{C}$ for $60 \mathrm{~min}$, the absorption maximum of the conjugates shifted hypsochromically. In case of conjugate 1 a large shift by $52 \mathrm{~nm}$ to $\lambda_{\max }=362 \mathrm{~nm}$ occurred whereas the maxima of conjugates $2-$ 5 only shifted by approximately $10 \mathrm{~nm}\left(\lambda_{\max }=406 \pm 4 \mathrm{~nm}\right)$. These blue-shifts are indicative of $\mathrm{H}$-type-like aggregation of the quaterthiophene moieties, in which the $\pi-\pi^{*}$ transition dipoles of the chromophores are mainly in a face-to-face alignment. Furthermore, the spectra of conjugates 2-5 in $\mathrm{THF} / \mathrm{H}_{2} \mathrm{O}$ display vibrational structures that are typical for $\mathrm{H}$-type aggregates of quaterthiophene moieties (Fig. 2). ${ }^{6,31}$

Circular dichroism (CD) spectra of conjugates 2-5 in THF : $\mathrm{H}_{2} \mathrm{O}(10: 90)$ exhibit positive bisignated Cotton effects (Fig. 2b-e, bottom, solid lines), indicating right-handed ( $P$-helical) chiral arrangements of the $\pi$-stacked chromophores. The zero-crossings of the bisignated bands of conjugates 2-5 correlate well with the maxima of absorbance in the UV/Vis spectra, which evidences the exciton-coupled character of these transitions. ${ }^{32}$ Notably, the spectrum of conjugate 3 differs from that of $\mathbf{2 , 4}$, and 5 as the ratio between the integrals of the positive and negative curves is more than twice as much ( $\sim 1.5: 1$ for $2 ; \sim 1: 1$ for 4 , and $5 ; \sim 3: 1$ for 3 ), indicating that the molecular organization of self-assembled 3 also differs. The CD spectrum of monomeric conjugate 1 also exhibits a Cotton effect. However, here the Cotton effect is significantly smaller than those of conjugates $\mathbf{2 - 5}$ and only visible when using a longer path length $(10 \mathrm{~mm})$ cuvette (Fig. 2a, solid lines). These spectroscopic findings demonstrate that 1-5 assemble in solutions of $\mathrm{THF} / \mathrm{H}_{2} \mathrm{O}$ into chiral aggregates. They also suggest that
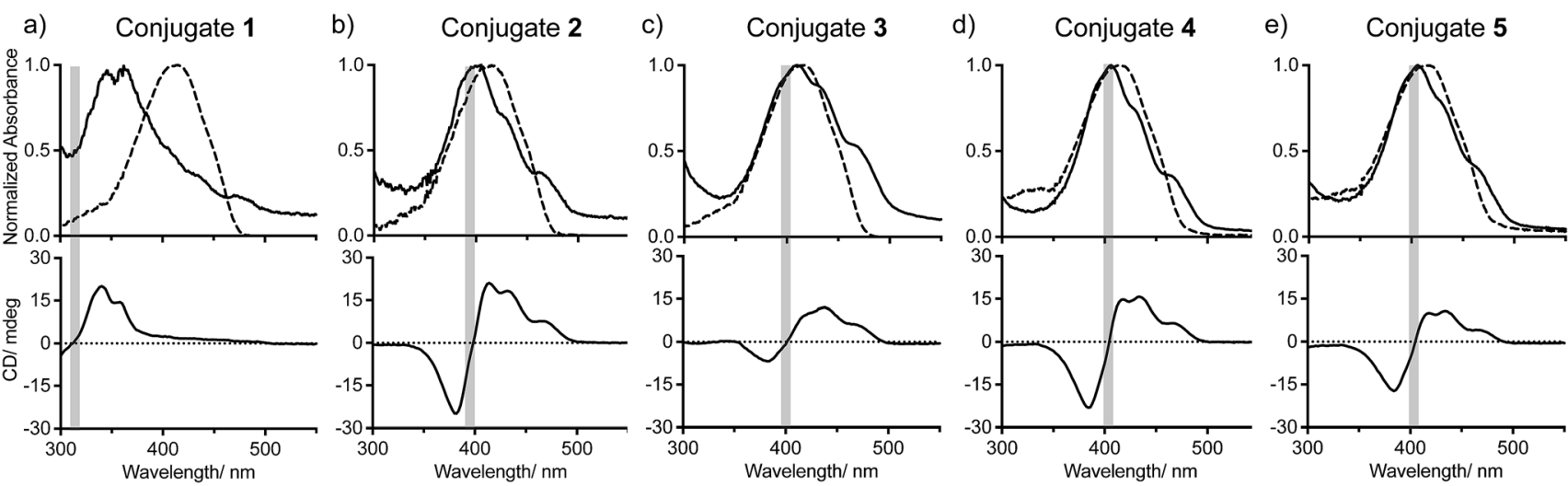

Fig. 2 UV/vis and CD spectra of conjugates 1 (a), 2 (b), 3 (c), 4 (d), and 5 (e) in THF (dashed lines) and $10: 90$ THF : $\mathrm{H}_{2} \mathrm{O}$ (solid lines). 
a) Conjugate 1

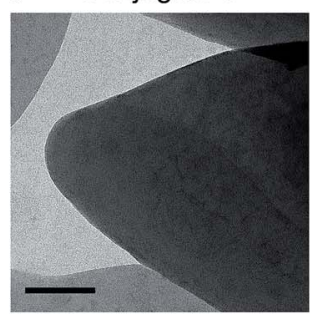

b) Conjugate 2

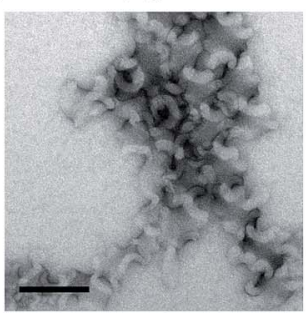

c) Conjugate 3

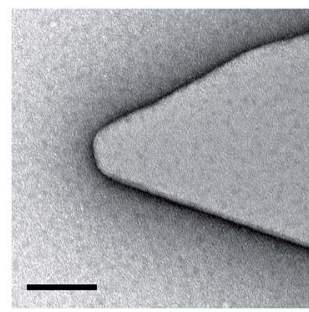

d)

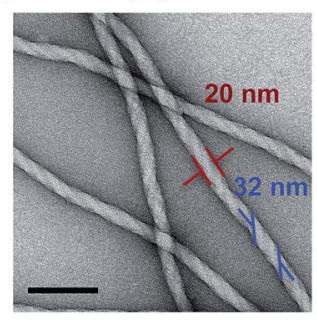

e) Conjugate 5

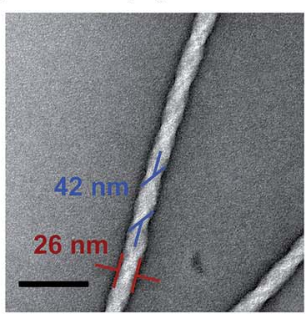

Fig. 3 TEM micrographs of conjugates 1 (a), 2 (b), 3 (c), 4 (d), and 5 (e) deposited from THF : $\mathrm{H}_{2} \mathrm{O} 20: 80$ after annealing at $90{ }^{\circ} \mathrm{C}$ for one hour and cooling. Scale bars represent $100 \mathrm{~nm}$.

conjugate 1 has a significantly different molecular organization in solution than conjugates $\mathbf{2 - 5}$.

\section{Self-assembly of oligoproline-quaterthiophene conjugates 1- 5 in the solid state}

Transmission electron microscopy. We used transmission electron microscopy (TEM) to investigate the morphology of the supramolecular assemblies formed by conjugates 1-5. The annealed solutions of 1-5 in THF : $\mathrm{H}_{2} \mathrm{O} 20: 80$ were deposited on carbon-coated copper grids and negatively stained with uranyl acetate. $\|$ The electron micrographs revealed distinct nanostructures dependent on the length of the oligoproline (Fig. 3). Surprisingly, despite the large difference in the UV/Vis and CD spectra, conjugates $\mathbf{1}$ and $\mathbf{3}$ form sheets with sizes extending into the micrometer regime (Fig. $3 \mathrm{a}$ and c, ESI $\dagger$ ). AFM studies revealed heights of $\sim 3.45 \mathrm{~nm}$ and $\sim 5.5 \mathrm{~nm}$ for the sheets formed by 1 and $\mathbf{3}$, respectively (ESI $\dagger$ ). These pronounced differences suggest different molecular self-assembly modes of the conjugates. TEM micrographs of self-assembled conjugate 2 show twirls that arise from tapes, which are curled up at the edges (Fig. 3b). AFM images of these kale-like structures displayed average heights of $\sim 3.4 \mathrm{~nm}$, which is similar to the sheets formed by 1 .

In contrast, the electron micrographs of nonamer $\mathbf{4}$ and dodecamer $\mathbf{5}$ furnish abundant nanofibrils consistently exceeding micrometer lengths with average widths of $20 \pm 1 \mathrm{~nm}$ and $26 \pm 2 \mathrm{~nm}$, respectively (Fig. $3 \mathrm{~d}$ and e, red). The TEM images also revealed that these fibrils are helical and have a right-handed twist. The average width of the twisted tape-like structures is $32 \pm 3 \mathrm{~nm}$ for $\mathbf{4}$ and $42 \pm 4 \mathrm{~nm}$ for $\mathbf{5}$, respectively (Fig. $3 \mathrm{~d}$ and e, blue). The ratios of the fiber widths $(20 \mathrm{~nm}: 26$ $\mathrm{nm})$ and tape widths (32 nm : $42 \mathrm{~nm}$ ) observed in the assemblies formed by 4 and 5 are approximately $9: 12$. This ratio coincides with the respective peptide lengths in both conjugates (9- and 12-mers) and indicates a relationship between the nanoscopic and molecular dimensions. The longer the oligoproline moiety is, the thicker is the helically twisted nanofibril.

Wide-angle X-ray scattering. To study the intriguing morphological differences between the shorter conjugates 1-3 and the longer analogues $\mathbf{4}$ and $\mathbf{5}$ at a molecular level we investigated conjugates 1-5 by means of grazing incidence wide-angle X-ray scattering (GIWAXS) (Fig. 4 and 5). The GIWAXS patterns for monomer $\mathbf{1}$ and hexamer $\mathbf{3}$ display a large number of distinct and sharp reflections, a signature of highly ordered supramolecular structures (Fig. 4a and 5a). The GIWAXS patterns for conjugates 2, 4, and 5, however, possess fewer distinct and sharp reflections in the thin film, evidencing a broader azimuthal distribution of the material. 9

The diffraction data for conjugates $\mathbf{1}$ and $\mathbf{3}$ are clearly different, a finding that corroborates the UV/Vis and CD results and proposes a different molecular organization depending on the oligoproline length. In the case of conjugate 1, the diffraction pattern revealed a wide-angle, equatorial scattering intensity (A) that is related to the typical $\pi$-stacking distance of $0.32 \mathrm{~nm}$ between chromophores (Fig. $4 \mathrm{a}$ and b). Additional welldefined scattering maxima (B) located at the middle-angle range support an intra-lamellar molecular order perpendicular to the $\pi-\pi$ stacking direction, with a $d$-spacing of $0.92 \mathrm{~nm}$ between molecules. Scattering maxima located at the meridional plane (C) are also indicative of a lamellar structure and denote a defined interlayer distance of $3.50 \mathrm{~nm}$.

Thus, GIWAXS of monomer $\mathbf{1}$ is in agreement with a face-toface assembly of the quaterthiophene moieties into sheets (Fig. 4b). An anti-parallel arrangement with the hydrophilic proline residues of alternating conjugates pointing up and down leads to sheets with a hydrophilic surface that shields the hydrophobic interior from the aqueous environment. The observed interlayer distance of $3.50 \mathrm{~nm}$ is in excellent agreement with the height of $\sim 3.45 \mathrm{~nm}$ observed by AFM. This value is also in accord with the molecular length of $3.37 \mathrm{~nm}$ reported for $\alpha, \alpha^{\prime}$-dihexylquaterthiophene. ${ }^{33}$ These findings suggest that the quaterthiophene with only one proline residue (1) creates a similar supramolecular organization as pure $\alpha, \alpha^{\prime}-$ dihexylquaterthiophene. ${ }^{34}$

The position and shape of GIWAXS reflections of trimer 2 suggest similar self-assembly properties as for the monomer, which comes as no surprise in view of the similar sheet heights found by AFM. However, these reflections do not provide sufficient information to precisely determine the molecular arrangement in the thin film. $\uparrow$ The azimuthally isotropic distribution indeed points toward a polycrystalline nature of the domains.

The GIWAXS pattern of hexamer 3 shows sharp distinguishable reflections $\left(\mathrm{B}^{\prime}\right)$ located at the equatorial plane, which points toward an ordered in-plane molecular structure with a $d$ spacing of $0.67 \mathrm{~nm}$ (Fig. 5a). Additional broad scattering maxima $\left(\mathrm{A}^{\prime}\right)$ located off the meridional plane and denoting a $d$ - 
a)

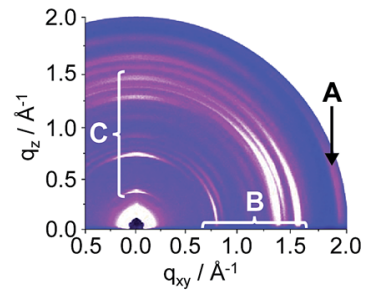

b)

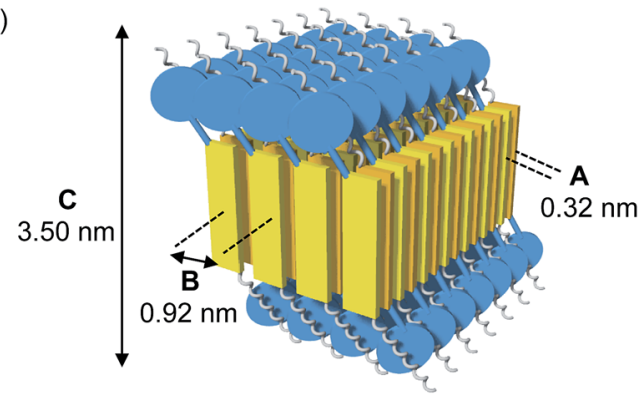

Fig. 4 (a) GIWAXS of conjugate 1. (b) Model of the mono-layered superstructure formed by 1 in the solid state. Blue disks represent proline residue, yellow plates represent quaterthiophene moieties, silver corkscrews represent alkyl chains.

a)

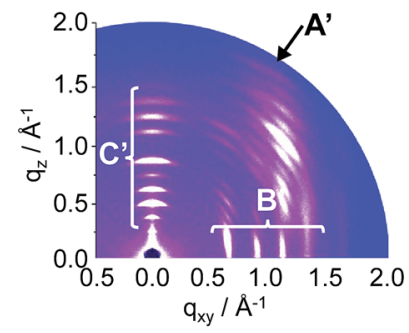

b)

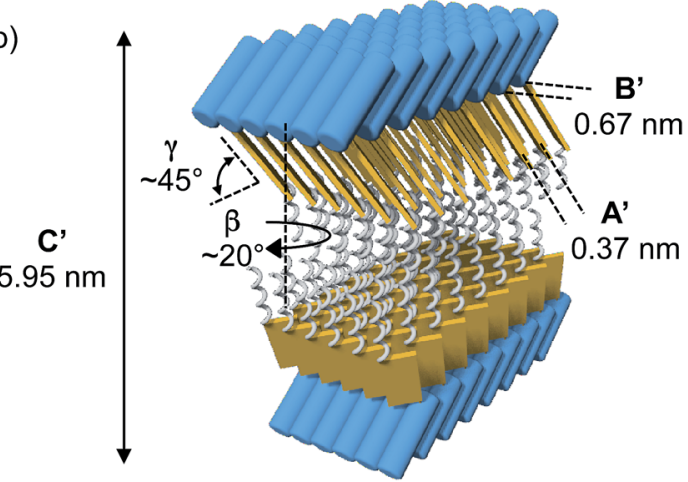

Fig. 5 (a) GIWAXS of conjugate 3. (b) Model of the double layered superstructure formed by 3 . Blue tubes represent oligoproline moieties, yellow plates represent quaterthiophene moieties, silver corkscrews represent alkyl chains.

spacing of $0.37 \mathrm{~nm}$ reveal $\pi-\pi$ stacking and suggest significant tilting $\left(\gamma \sim 45^{\circ}\right)$ and rotation $\left(\beta \sim 20^{\circ}\right)$ of the chromophores relative to the in-plane layer. The GIWAXS also displays scattering maxima at the meridional plane $\left(\mathrm{C}^{\prime}\right)$ suggesting a lamellar structure with an interlayer distance of $5.95 \mathrm{~nm}\left(\mathrm{C}^{\prime}\right.$, Fig. 5a). This value is in good agreement with the height measured by AFM $(\sim 5.5 \mathrm{~nm})$ and almost twice the estimated height of conjugate 3 . One can thus conclude that the conjugate exists in a double layer with the hydrophobic $\pi-\pi$ stacked quaterthiophene moieties in the interior and the hydrophilic oligoprolines exposed to the solvent (Fig. 5b).

Within the single layers, the quaterthiophenes are in a parallel arrangement with the oligoprolines on the same side. This molecular organization of conjugate 3 differs significantly from that of conjugate 1 . In case of conjugate 1 , the small monomeric proline residue allows for $\pi-\pi$ interactions of antiparallel arranged building blocks, which result in the formation of a single layer. In case of conjugate 3 such an anti-parallel arrangement is obstructed by the hexaproline moiety. The longer proline hexamer enforces a parallel arrangement of the conjugate. As a result single layers with a hydrophobic and a hydrophilic face are formed. These owe their stability to an assembly into a double layer in which the hydrophilic oligoproline surfaces shield the hydrophobic interior from the aqueous environment. Thus, the length of the oligoproline has a direct bearing on the molecular arrangement of the conjugates. It is noteworthy that both conjugates $\mathbf{1}$ and $\mathbf{3}$ assemble into sheets but with intrinsically different molecular structures.

The GIWAXS patterns for both the nonamer 4 and dodecamer 5 display broad reflections with low intensities that indicate lower molecular order as compared to that observed for conjugates 1 and 3.§ Such patterns are expected for helical structures, which are more difficult to visualize by GIWAXS compared to sheets. Reassuringly, the position and shape of the visible reflections support a hexagonal arrangement of the aggregates, which is a common feature seen for columnar structural motifs. ${ }^{35}$ The position of the reflections at the small angle range indicate unit cells with $a_{\mathrm{hex}} \sim 2.7 \mathrm{~nm}$ for conjugates 4 and 5. This value is smaller than the length of the conjugates ( $\sim 3$ and $4 \mathrm{~nm}$, respectively) and the diameter of the threads in the TEM images (Fig. 3d and e), which suggests a more complex, likely helical arrangement of the quaterthiophene conjugates. This rationale is in agreement with the twist that is visible in the TEM images. In comparison to conjugate 3 , which comprises a hexaproline moiety, the nona- and dodecaproline moieties in conjugates $\mathbf{4}$ and $\mathbf{5}$ may generate an irregularity in the doublelayered sheet. The molecules would thus rotate along the $\pi-\pi$ stacking direction, causing the formation of ribbons arranged in a spiral. This would explain the unusually small $d$-spacing between chromophores of approximately $3 \mathrm{~nm}$. Additionally, the longer oligoprolines, unlike the shorter hexaproline can shield the hydrophobic quaterthiophene interior from water in the twisted, helical ribbons, and do not require packing of the oligoproline into flat sheets.**

\section{Conclusions}

In conclusion, we have demonstrated how the molecular assembly of quaterthiophene moieties, which have a pronounced intrinsic tendency toward aggregation, changes by attaching oligoprolines. It should be noted that the latter do not self-assemble on their own. Varying the length of the oligoproline moieties caused a substantial change in the molecular organization of the conjugates from monolayers to double- 
layered sheets and helically twisted ribbons. The dimensions observed for the nanostructures correlate with the molecular dimensions of the building blocks. This agreement of length scales observed at the molecular and the supramolecular level, which is not commonly observed, allows for tuning of the supramolecular architectures. Thus, the results provide guidelines for the rational design of related conjugates with predictable self-assembly properties. In addition, prior studies with oligoproline-perylene monoimide conjugates had provided supramolecular assemblies with unique molecular arrangements. ${ }^{21-23}$ The present study thus suggests to target triades comprising perylene chromophores, oligothiophenes, and the oligoproline scaffold as this design creates a combination of electron-donor and electron-acceptor components.

\section{Conflicts of interest}

There are no conflicts to declare.

\section{Acknowledgements}

We are grateful for financial support by the VW foundation and support by the ETH Scientific Center for Optical and Electron Microscopy (ScopeM). We thank Dr Elena Mena-Osteritz (University of Ulm) for insightful comments. We also gratefully acknowledge beamline 9 of the DELTA electron storage ring in Dortmund for providing synchrotron radiation and technical support for the GIWAXS measurements.

\section{Notes and references}

$\S$ We also prepared and studied the respective amide analogue of conjugate 1 . The optical properties and TEM micrographs of this conjugate 1a are very similar to those of 1, however, the observed GIWAXS reflections are not nearly as well resolved as those of $\mathbf{1}$ (see the ESI $\dagger$ ). These findings suggest that the self-assembly of $\mathbf{1 a}$ is very similar as that of $\mathbf{1}$ but the aggregates are less crystalline.

I For details, see the ESI. $\dagger$

\| A THF : $\mathrm{H}_{2} \mathrm{O}$ mixture of $20: 80$ was used for TEM analyses since in mixtures of 10 : 90 complete precipitation was observed for some of the conjugates, which rendered their deposition on the TEM grids difficult. Spot checks showed that the same supramolecular structures were formed in THF : $\mathrm{H}_{2} \mathrm{O}$ mixture of $20: 80$ and 10 : 90; see the ESI $\dagger$ for details. Noteworthy, the self-assemblies form in solution upon annealing as evidenced, in some cases even, by visual inspection.

** This conclusion is supported by the observation that upon increasing the amount of water in the solutions of conjugates 4 and 5 to $100 \%$ water, the TEM of these structures show curled sheets instead of twisted fibers (see the ESI $\dagger$ ). Upon the addition of so much water, it seems that even the longest oligoprolines could not fully shield the interior quaterthiophenes without becoming more sheet-like.

1 Handbook of Oligo- and Polythiophenes, ed. D. Fichou, Wiley VCH, Weinheim, Germany, 1999.

2 (a) J. Roncali, Chem. Rev., 1997, 97, 173-205; (b) G. Barbarella, M. Melucci and G. Sotgiu, Adv. Mater., 2005, 17, 1581-1593; (c) G. Barbarella, M. Zambianchi, O. Pudova, V. Paladini, A. Ventola, F. Cipriani, G. Gigli, R. Cingolani and G. Citro, J. Am. Chem. Soc., 2001, 47, 11600-11607.

3 A. Mishra and P. Bäuerle, Angew. Chem., Int. Ed., 2012, 51, 2020-2067.
4 P. Leclère, M. Surin, P. Viville, R. Lazzaroni, A. F. M. Kilbinger, O. Henze, W. J. Feast, M. Cavallini, F. Biscarini, A. P. H. J. Schenning and E. W. Meijer, Chem. Mater., 2004, 16, 4452-4466.

5 X. Lin, M. Suzuki, M. Gushiken, M. Yamauchi, T. Karatsu, T. Kizaki, Y. Tani, K.-i. Nakayama, M. Suzuki, H. Yamada, T. Kajitani, T. Fukushima, Y. Kikkawa and S. Yagai, Sci. Rep., 2017, 43098.

6 S. B. Dkhil, M. Pfannmöller, I. Ata, D. Duché, M. Gaceur, T. Koganezawa, N. Yoshimoto, J.-J. Simon, L. Escoubas, C. Videlot-Ackermann, O. Martgeat, S. Bals, P. Bäuerle and J. Ackermann, J. Mater. Chem. A, 2017, 5, 1005-1013.

7 Z. Guo, Y. Song, R. Gong, Y. Mu, Y. Jiang, M. Li and X. Wan, Supramol. Chem., 2014, 26, 383-391.

8 G. M. Whitesides and B. Grzybowski, Science, 2002, 295, 2418-2421.

9 H.-A. Klok, A. Rösler, G. Götz, E. Mena-Osteritz and P. Bäuerle, Org. Biomol. Chem., 2004, 2, 3541-3544.

10 W.-W. Tsai, L.-s. Li, H. Cui, H. Jiang and S. I. Stupp, Tetrahedron, 2008, 64, 8504-8514.

11 R. J. Kumar, J. M. MacDonald, T. B. Singh, L. J. Waddington and A. B. Holmes, J. Am. Chem. Soc., 2011, 133, 8564-8573.

12 H. A. M. Ardoña and J. D. Tovar, Bioconjugate Chem., 2015, 26, 2290-2302.

13 D. A. Stone, L. Hsu and S. I. Stupp, Soft Matter, 2009, 5, 19901993.

14 K. Besar, H. A. M. Ardoña, J. D. Tovar and H. E. Katz, ACS Nano, 2015, 9, 12401-12409.

15 S. R. Diegelmann, J. M. Gorham and J. D. Tovar, J. Am. Chem. Soc., 2008, 130, 13840-13841.

16 E. K. Schillinger, E. Mena-Osteritz, J. Hentschel, H. G. Börner and P. Bäuerle, Adv. Mater., 2009, 21, 1562-1567.

17 L. Tian, R. Szilluweit, R. Marty, L. Bertschi, M. Zerson, E.-C. Spitzner, R. Magerle and H. Frauenrath, Chem. Sci., 2012, 3, 1512-1521.

18 H. A. M. Ardoña, E. R. Draper, F. Citossi, M. Wallace, L. C. Serpell, D. J. Adams and J. D. Tovar, J. Am. Chem. Soc., 2017, 139, 8685-8692.

19 E.-K. Schillinger, M. Kümin, A. Digennaro, E. Mena-Osteritz, S. Schmid, H. Wennemers and P. Bäuerle, Chem. Mater., 2013, 25, 4511-4521.

20 A. Digennaro, H. Wennemers, G. Joshi, S. Schmid, E. MenaOsteritz and P. Bäuerle, Chem. Commun., 2013, 49, 1092910931.

21 (a) U. Lewandowska, W. Zajaczkowski, L. Chen, F. Bouillière, D. Wang, K. Koynov, W. Pisula, K. Müllen and H. Wennemers, Angew. Chem., Int. Ed., 2014, 53, 1253712541; (b) U. Lewandowska, W. Zajaczkowski, W. Pisula, Y. Ma, C. Li, K. Müllen and H. Wennemers, Chem.-Eur. J., 2016, 22, 3804-3809.

22 U. Lewandowska, W. Zajaczkowski, S. Corra, J. Tanabe, R. Borrmann, E. M. Benetti, S. Stappert, K. Watanabe, N. A. K. Ochs, R. Schaeublin, C. Li, E. Yashima, W. Pisula, K. Müllen and H. Wennemers, Nat. Chem., 2017, 9, 1068.

23 U. Lewandowska, S. Corra, W. Zajaczkowski, N. A. K. Ochs, M. S. Shoshan, J. Tanabe, S. Stappert, C. Li, E. Yashima, 
W. Pisula, K. Müllen and H. Wennemers, Chem.-Eur. J, 2018, 24, 12623-12629.

24 F. Rabanal, M. D. Ludevid, M. Pons and E. Giralt, Biopolymers, 1993, 33, 1019-1028.

25 S. Kakinoki, Y. Hirano and M. Oka, Polym. Bull., 2005, 53, 109-115.

26 L. Garbuio, B. Lewandowski, P. Wilhelm, L. Ziegler, M. Yulikov, H. Wennemers and G. Jeschke, Chem.-Eur. J., 2015, 21, 10747-10753.

27 P. Wilhelm, B. Lewandowski, N. Trapp and H. Wennemers, J. Am. Chem. Soc., 2014, 136, 15829-15832.

28 L. Stryer and R. P. Haugland, Proc. Natl. Acad. Sci. U. S. A., 1967, 58, 719-726.

29 S. Dobitz, M. R. Aronoff and H. Wennemers, Acc. Chem. Res., 2017, 50, 2420-2428.

30 J. Wan, A. Ferreira, W. Xia, C. H. Chow, K. Takechi, P. V. Kamat, G. Jones and V. I. Vullev, J. Photochem. Photobiol., A, 2008, 197, 364-374.
31 M. Pope and C. E. Swenberg, Electronic Processes in Organic Crystals, Clarendon Press, Oxford, 1982.

32 N. D. Harada and K. Nakanishi, Circular dichroic spectroscopy: exciton coupling in organic stereochemistry, University Science Books, 1983.

33 A. Facchetti, M. Mushrush, M. H. Yoon, G. R. Hutchison, M. A. Ratner and T. J. Marks, J. Am. Chem. Soc., 2004, 126, 13859-13874.

34 F. Garnier in, Electronic Materials: The Oligomer Approach, ed. K. Müllen and G. Wegner, Wiley-VCH, Weinheim, 1998, pp. 559-583.

35 (a) X. Feng, W. Pisula, M. Takase, X. Dou, V. Enkelmann, M. Wagner, N. Ding and K. Müllen, Chem. Mater., 2008, 20, 2872-2874; (b) W. Pisula, Z. Tomovic, M. D. Watson, K. Müllen, J. Kussmann, C. Ochsenfeld, T. Metzroth and J. Gauss, J. Phys. Chem. B, 2007, 111, 7481-7487. 